\section{JTI}

JOURNAL OF

TRAUMA AND INJURY

\title{
Delayed Subclavian Vein Stenosis without Thrombosis Following Clavicle Fracture
}

\author{
Do Wan Kim, M.D. ${ }^{1}$, In Seok Jeong, M.D. ${ }^{1}$, Kook Joo Na, M.D. ${ }^{2}$ \\ ${ }^{1}$ Department of Thoracic and Cardiovascular Surgery, Chonnam National University \\ Hospital, Chonnam National University Medical School, Gwangju, Korea \\ ${ }^{2}$ Department of Thoracic and Cardiovascular Surgery, Chonnam National University \\ Hwasun Hospital, Chonnam National University Medical School, Hwasun, Korea
}

Received: August 1, 2019

Revised: November 26, 2019

Accepted: December 4, 2019

\section{Correspondence to}

Kook Joo Na, M.D.

Department of Thoracic and Cardiovascular Surgery, Chonnam National University Hwasun Hospital, Chonnam National University Medical School, 322 Seoyang-ro, Hwasun-eup, Hwasun 58128, Korea

Tel: +82-61-379-7664

Fax: +82-61-379-7665

E-mail: kjna@chonnam.ac.kr
Subclavian vein injuries occasionally occur as a sequela of penetrating trauma or vascular access, but have rarely been reported to occur after clavicle fracture. The subclavian vessels are mainly enclosed by the subclavius muscle, the first rib, and the costocoracoid ligament. Therefore, in such cases, subclavian vein injury is rare because of the strcutures surrounding the subclavian vessels. Nevertheless, subclavian vein injuries occasionally show thrombotic manifestations, and thrombosis of the upper limbs constitutes $1-4 \%$ of cases of total deep vein thrombosis. Furthermore, to the best of the authors' knowledge, although vessel injuries have been reported after clavicle or rib fractures and nerve injuries to regions such as the brachial plexus, no case involving delayed presentation of isolated subclavian vein stenosis after clavicle fracture due to blunt trauma has yet been reported.

Keywords: Subclavian vein; Clavicle; Vascular system injuries

\section{INTRODUCTION}

The major mechanical complications of subclavian vein injuries can be divided into stenosis and thrombotic occlusion, with the exception of free perforation. Subclavian vein stenosis most commonly occurs as a complication of hemodialysis device insertion via the subclavian vein or internal jugular vein and transvenous pacemaker implantation [1-3]. The incidence of central venous catheterization-related thrombosis is $2 \%$ to $6 \%$, and making it an uncommon complication [4,5]. Furthermore, subclavian vessel injuries caused by blunt trauma can occur in either the artery or vein, but stud- 
ies have mainly focused on arterial rupture [6]. Here, we report a case involving delayed presentation of isolated subclavian vein stenosis after clavicle fracture due to blunt trauma.

\section{CASE REPORT}

A 62-year-old man was transferred to Chonnam National Univeristy Hospital after a traffic accident for the treatment of severe trauma. The patient had a 3-month history of hyperlipidemia. His arterial systemic blood pressure was 140/90 $\mathrm{mmHg}$, his heart rate was 84 beats/min, his respiratory rate was 24 breaths/min, and his body temperature was $36^{\circ} \mathrm{C}$. Chest and abdominal computed tomography (CT) scans were obtained, and showed bilateral multiple rib fractures, hemopneumothorax, lung contusion, and an inserted left chest tube. Additionally, type B aortic dissection and liver contusion were confirmed (Fig. 1). The fracture of the right clavicle body and the posterior wall of the right acetabulum, as well as an open fracture of the right tibio-fibula, were discovered on radiography (Fig. 2). The abbreviated injury scale was five points in the chest due to the major aortic injury, three points in the extremities due to the open right tibia fracture, and two points in the abdomen due to the liver contusion; therefore, the injury severity score was 38 points. Before performing thoracic endovascular aortic repair (TEVAR), right empyema developed, and right thoracic decortication commenced on the 14th day after admission (Fig. 3). To treat the open tibia fracture, 25 days after admission, external fixation was done at the fracture site, and after control of a general infection, TEVAR was performed on the 43rd day after admission. The patient was discharged after 75 days in the
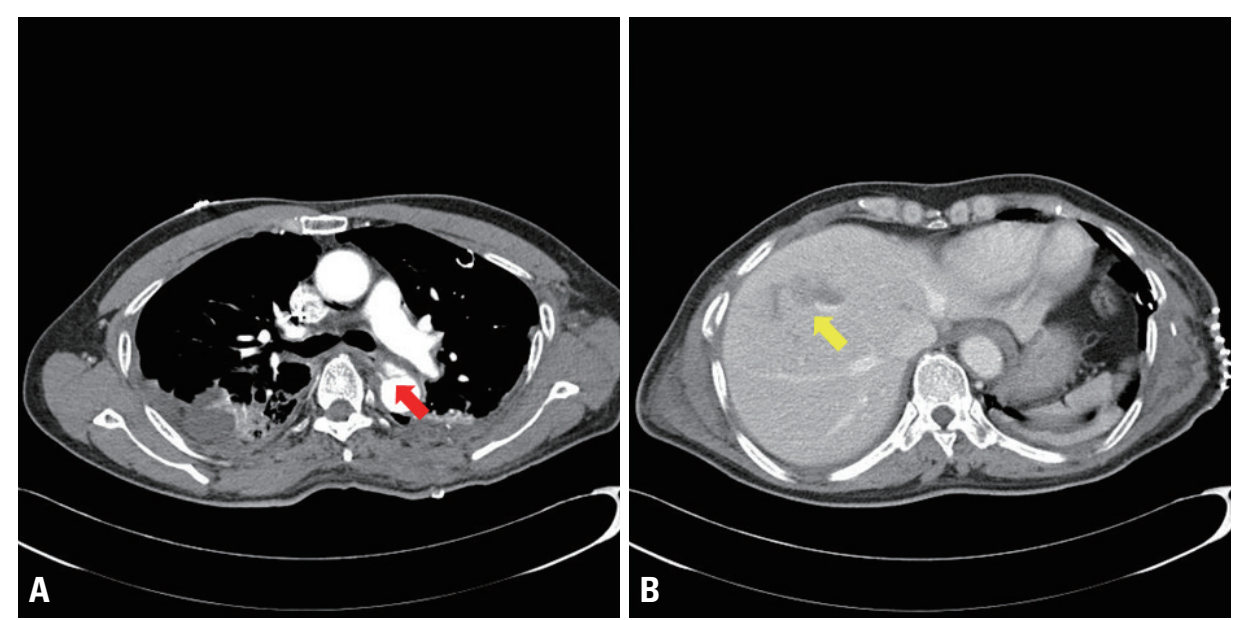

Fig. 1. Computed tomography scan at the initial diagnosis. (A) Traumatic aortic dissection (arrow). (B) Liver contusion (arrow).
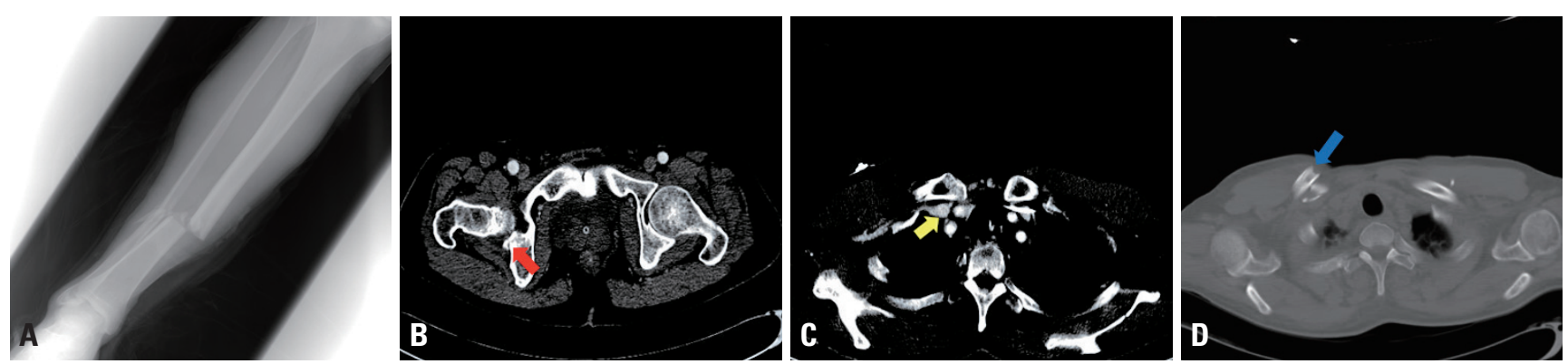

Fig. 2. Accompanying injuries after trauma at the initial diagnosis. (A) Right tibio-fibular open fracture. (B) Right acetabular posterior wall fracture (arrow). (C) Right subclavian vein without stenosis below the clavicle fracture site (arrow). (D) Right clavicle fracture (arrow). 
hospital following rehabilitation with long-term bed rest, which resulted in bone union, so no definitive surgery was performed. Regarding the clavicle fracture, we consulted a team of orthopedic surgeons, who concluded that the patient did not have functional problems; therefore, conservative treatment was chosen instead of surgery. The patient was lost to follow-up after discharge until 8 years later, when the patient was admitted to the emergency department with abdominal pain. A gallbladder stone was the cause of abdominal pain, and chest CT angiography was performed to check the current status of TEVAR and the previous chest trauma lesion. On this examination, the diameter of the right subclavian vein was found to be reduced by about $80 \%$, and a number of collateral branches had formed around the right subclavian vein (Fig. 4). To summarize, there was no stenosis at the time of initial admission for trauma, and right subclavian vein stenosis was found 114 months after the trauma. Currently, the patient has stable activity without arm swelling or pain.
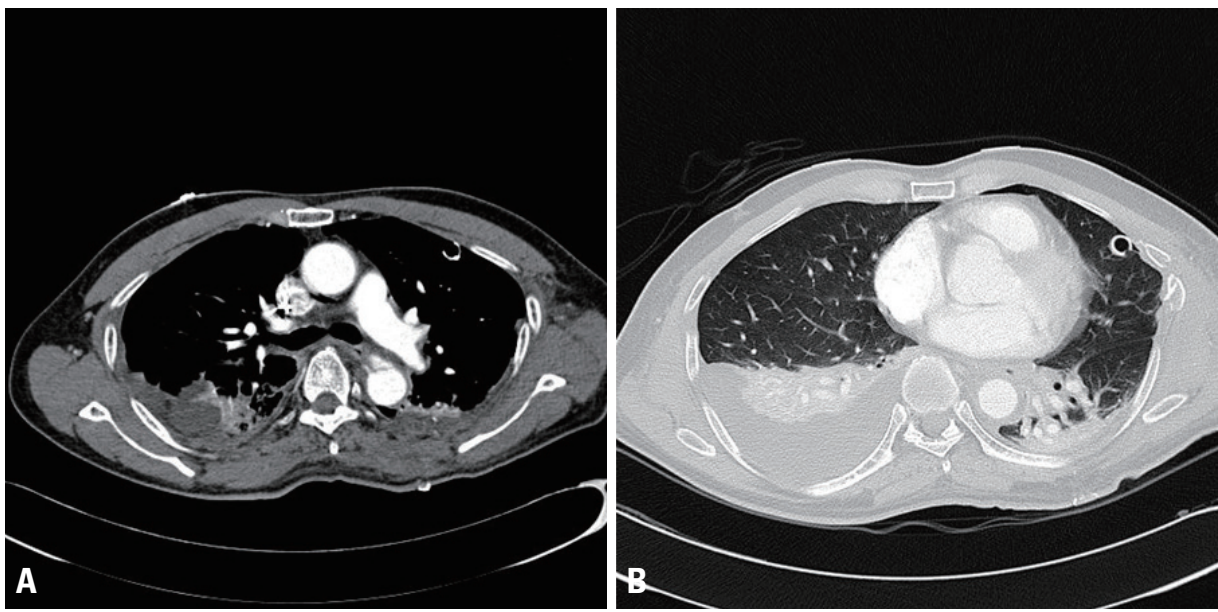

Fig. 3. Chest computed tomography of right empyema. (A) Right thoracic cavity multifocal empyema with traumatic aortic dissection (enhanced setting). (B) Right thoracic cavity empyema (lung setting).
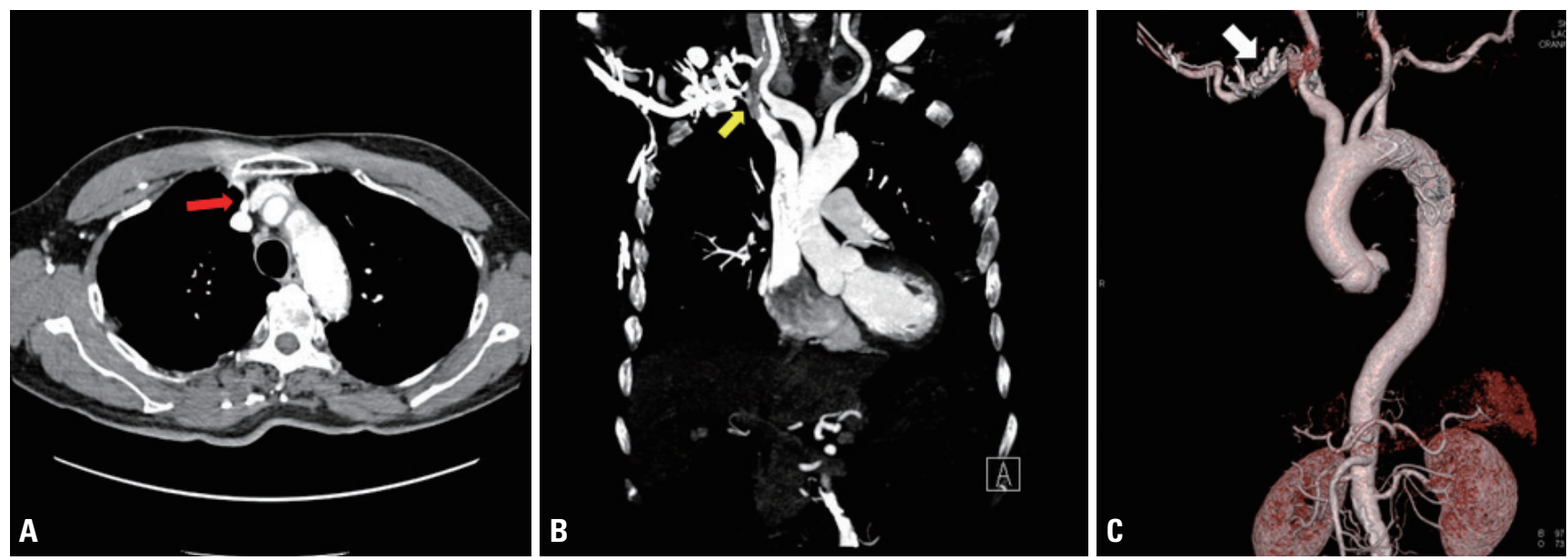

Fig. 4. Chest computed tomography of subclavian vein stenosis at an 8-year follow-up. (A) Right subclavian vein stenosis (transverse view, arrow). (B) Right subclavian vein stenosis (coronal view, arrow). (C) Right subclavian vein with collateral branches (three-dimensional reconstruction view, arrow). 


\section{DISCUSSION}

Clavicle fractures are common after bone injuries, of which midshaft fracture is the most common type after blunt trauma [7]. However, subclavian vessel injuries caused by clavicle fracture are uncommon, because the perivascular tissues of the subclavian vessels are tightly protected by muscles, ligaments, and ribs [6]. Subclavian artery injuries are known to be potentially fatal, and are therefore the main treatment target in patients with subclavian vessel injuries. In contrast, the complications of venous injuries-including stenosis and thrombotic occlusion-are less serious, although cases of free perforation can be more serious. Isolated subclavian venous injuries are occasionally not detected through physical symptoms, whereas subclavian artery stenosis causes upper limb ischemia. Therefore, in cases with no arterial injuries in the acute phase, venous injuries are difficult to diagnose, and low venous pressure often results in the cessation of spontaneous bleeding [8]. Nonetheless, some studies with a small number of patients have reported the proportion of venous injuries that occur in cases of trauma [9].

The treatment modalities for subclavian vein injuries vary depending on the injury type (e.g., free perforation, stenosis, or thrombotic occlusion). It is justifiable for the subclavian vein to be ligated for damage control surgery in unstable patients. In situations other than damage control, revascularization can be done via either an open surgical approach (primary repair or bypass surgery with an autologous/artificial graft, with or without thrombectomy) or an endovascular approach (stent graft insertion with or without thrombectomy) [10]. However, surgical thrombectomy alone has been reported in patients with subclavian vein thrombosis and clavicle fracture, with symptoms including upper limb swelling and pain [11].

Studies have also investigated central venous stenosis in patients in whom a catheter has been inserted for dialysis due to kidney failure [11]. Several theories have been suggested regarding the mechanism by which subclavian vein stenosis occurs, with principal proposals including thrombosis, compromised venous return in dialysis [12]. In addition, fibrosis and keloids are known to be involved [13]. Anatomically, the proximal site of the subclavian vein is compressed at the costoclavicular ligament and subclavius tendon $[1,3,12]$. However, in the traumatic field, the cause of venous stenosis is may be unclear. Anticoagulation therapy is necessary for patency after revascularization. When there are no serious symptoms relating to subclavian vein stenosis/occlusion, such as arm swelling or pain, revascularization may not be necessary. In such situations, anticoagulation therapy can be done for cases of thrombotic occlusion [12].

Although clavicle fractures are a commonly-encountered type of injury, intermittent delayed complications such as vessel injury and thrombosis have been reported in some patients $[8,11,14]$. Previous studies have been shown that upper limb ischemia can be caused by external compression of vascular injuries, hematomas, and vessel structure after fracture [5-7,14]. In particular, external pressure and subsequent thrombosis are one of the main mechanisms underlying delayed vascular complications after clavicle fracture $[8,14]$. Currently, surgical treatment is superior to conservative treatment with respect to the success rate of union in clavicle fractures, and surgical fixation may be helpful if the displacement of a clavicle fracture is severe. The treatment approach needs to be tailored according to the individual [15]. For this patient, who was relatively young with good physical activity status, surgery should have been considered as a treatment option. However, since little research has been done on this topic, prospective large-scale studies of the surgical treatment of clavicle fractures will be needed. Based on our review of previous studies, the delayed presentation of subclavian vein stenosis after clavicle fracture due to blunt trauma has not yet been reported.

\section{REFERENCES}

1. Clark DD, Albina JE, Chazan JA. Subclavian vein stenosis and thrombosis: a potential serious complication in chronic hemodialysis patients. Am J Kidney Dis 1990;15:265-8.

2. Abu-El-Haija B, Bhave PD, Campbell DN, Mazur A, Hodgson-Zingman DM, Cotarlan V, et al. Venous stenosis after transvenous lead placement: a study of outcomes and risk factors in 212 consecutive patients. J Am Heart Assoc 2015;4:e001878.

3. Bakhshoude B, Ravari H, Kazemzadeh GH, Rad MP. Diagnostic 
value of computerized tomography venography in detecting stenosis and occlusion of subclavian vein and superior vena in chronic renal failure patients. Electron Physician 2016;8:2781-6.

4. Gentile A, Petit L, Masson F, Cottenceau V, Bertrand-Barat J, Freyburger G, et al. Subclavian central venous catheter-related thrombosis in trauma patients: incidence, risk factors and influence of polyurethane type. Crit Care 2013;17:R103.

5. Hill JM, McGuire MH, Crosby LA. Closed treatment of displaced middle-third fractures of the clavicle gives poor results. J Bone Joint Surg Br 1997;79:537-9.

6. Assenza M, Centonze L, Valesini L, Campana G, Corona M, Modini C. Traumatic subclavian arterial rupture: a case report and review of literature. World J Emerg Surg 2012;7:18.

7. Kihlström C, Möller M, Lönn K, Wolf O. Clavicle fractures: epidemiology, classification and treatment of 2422 fractures in the Swedish Fracture Register; an observational study. BMC Musculoskelet Disord 2017;18:82.

8. Cooney DR, Kloss B. Case report: delayed subclavian vein injury secondary to clavicular malunion. J Emerg Med 2012;43:648-50.
9. Akyuz M, Gokalp O, Ozcem B, Ozcan S, Besir Y, Gurbuz A. Surgical management of axillosubclavian vascular injuries. Pak J Med Sci 2015;31:552-5.

10. Giannakopoulos TG, Avgerinos ED. Management of peripheral and truncal venous injuries. Front Surg 2017;4:46.

11. Wright SW. Subclavian vein thrombosis following clavicular fracture. Emerg Med J 2009;26:840.

12. Dhakal P, Gundabolu K, Bhatt VR. An algorithmic approach to management of venous thromboembolism. Clin Appl Thromb Hemost 2017;23:511-7.

13. Barrett N, Spencer S, McIvor J, Brown EA. Subclavian stenosis: a major complication of subclavian dialysis catheters. Nephrol Dial Transplant 1988;3:423-5.

14. Ranke H, Märdian S, Haas NP, Baecker H. Thrombosis of the subclavian vein after conservative treatment of a clavicular fracture: a rare complication. Unfallchirurg 2016;119:255-8.

15. Ropars M, Thomazeau H, Huten D. Clavicle fractures. Orthop Traumatol Surg Res 2017;103:S53-9. 